\title{
Effect of tumor microenvironmental factors on tumor growth dynamics modeled by correlated colored noises with colored cross-correlation
}

\begin{abstract}
The effect of non-immunogenic tumor microenvironmental factors on tumor growth dynamics modeled by correlated additive and multiplicative colored noises is investigated. Using the Novikov theorem, Fox approach and Ansatz of Hanggi, an approximate FokkerPlanck equation for the system is obtained and analytic expression for the steady state distribution Pst(x) is derived. Based on the numerical results, we find that fluctuations of microenvironmental factors within the tumor site with parameter of have a diffusive effect on the tumor growth dynamics, and the tumor response to the microenvironmental factors with parameter Ŭ inhibits growth at weak correlation time Ü Moreover, at increasing correlation time Üthe inhibitive effect of tumor response Ǔis suppressed and instead a systematic growth promotion is noticed. The result also reveals that the strength of the correlation time Ühas a strong influence on the growth effects exerted by the non-immunogenic component of tumor microenvironment on tumor growth.
\end{abstract}

Keyword: Langevin equation; Fokkerï Planck equation; Colored noise; Tumor growth dynamics 